\title{
Pólen de Magnoliopsida (Asteridae) e Liliopsida do perfil sedimentar de uma turfeira em São Francisco de Paula, Planalto Leste do Rio Grande do Sul, Sul do Brasil ${ }^{1}$
}

\author{
ADRIANA LEONHARDT ${ }^{2,3}$ e MARIA LUISA LORSCHEITTER ${ }^{2}$
}

(recebido: 26 de agosto de 2009; aceito: 06 de maio de 2010)

\begin{abstract}
Magnoliopsida (Asteridae) and Liliopsida pollen of the sedimentary profile of a bog, São Francisco de Paula, Eastern Plateau of Rio Grande do Sul, Southern Brazil). This paper aims to provide data for paleopalynological research progress in southern Brazil by increasing the knowledge about the Quaternary in the region. Therefore, the palynological analysis of a sedimentary profile of a bog of Rio Grande do Sul Eastern Plateau was made and the Angiospermae pollen (Asteridae, Alismatidae, Commelinidae and Liliidae) are presented. The profile is $286 \mathrm{~cm}$ long, corresponding approximately to the last 25,000 years. The chemical processing of the 22 samples obtained followed the standard methodology and the analysis was made by light microscopy. The photomicrographs are accompanied by concise morphological descriptions, including measurements of polar and equatorial axis and ecological data of the original sporophyte. Pollen corresponding to 21 Asteridae, one Alismatidae, four Commelinidae and three Liliidae were identified, beside three indeterminate pollens of Angiospermae.
\end{abstract}

Key words - Angiospermae, palynology, Quaternary

RESUMO - (Pólen de Magnoliopsida (Asteridae) e Liliopsida do perfil sedimentar de uma turfeira em São Francisco de Paula, Planalto Leste do Rio Grande do Sul, Sul do Brasil). O trabalho tem por finalidade fornecer material de referência para pesquisas paleopalinológicas no sul do Brasil através da análise do perfil sedimentar de uma turfeira do Planalto Leste do Rio Grande do Sul. São apresentados os grãos de pólen de Asteridae, Alismatidae, Commelinidae e Liliidae. O perfil possui $286 \mathrm{~cm}$ de comprimento correspondendo, aproximadamente, aos últimos 25.000 anos. O processamento químico das 22 amostras obtidas seguiu o método padrão e a análise foi feita em microscopia óptica. As descrições morfológicas, incluindo medidas dos eixos polar e equatorial, são acompanhadas de fotomicrografias e dados ecológicos do esporófito de origem. Foram identificados grãos correspondentes a 21 Asteridae, uma Alismatidae, quatro Commelinidae, três Liliidae e mais três grãos de pólen indeterminados de Angiospermas.

Palavras-chave - Angiosperma, palinologia, Quaternário

\section{Introdução}

As mudanças climáticas e sua influência na vegetação dos últimos milênios influenciaram os padrões fitogeográficos da atualidade. A palinologia de sedimentos é uma ferramenta bastante útil na análise histórica da vegetação ao investigar processos ao longo do tempo geológico. Por possuir morfologia ligada geneticamente ao esporófito da espécie, as análises quantitativas de pólen depositado em sedimentos, associadas a datações radiométricas, possibilitam reconstituições paleoambientais. A confiabilidade dos resultados está, porém, diretamente relacionada a uma correta identificação botânica dos grãos de pólen, o

1. Parte da dissertação de mestrado da primeira autora, Programa de Pós-Graduação em Botânica, Universidade Federal do Rio Grande do Sul, RS, Brasil.

2. Universidade Federal do Rio Grande do Sul, Instituto de Biociências, Departamento de Botânica. Av. Bento Gonçalves 9500, 91540-000 Porto Alegre, RS, Brasil.

3. Autor para correspondência: adriana.leonhardt@yahoo.com.br que faz dos catálogos polínicos referências básicas em análise paleoambiental.

Na atualidade, o Planalto Leste do Rio Grande do Sul é composto por mata com araucária, campo e turfeiras (Rambo 1956). O clima da região, Mesotérmico Médio Superúmido (Nimer 1989), favorece a expansão florestal sobre o extenso campo de relevo ondulado (Veloso 1962, Klein 1975, Rambo 1953, 2000). Poucos trabalhos sobre palinologia de sedimentos foram desenvolvidos na região visando análise de paleoambientes (Roth \& Lorscheitter 1993, Behling et al. 2001, 2004), o que dificulta o entendimento dos processos relacionados à dinâmica da vegetação dos últimos milênios. Este trabalho tem, portanto, o objetivo de fornecer subsídios para novas pesquisas paleoambientais no Planalto Leste ao apresentar material básico de referência para identificação de grãos de pólen preservados em sedimentos. O material é proveniente de um perfil sedimentar de $286 \mathrm{~cm}$ em uma turfeira atual da região, situada em Alpes de São Francisco. O intervalo de tempo compreende os últimos 25.000 anos (final do Pleistoceno e todo o Holoceno), 
conforme a datação radiométrica realizada na base do perfil.

Este trabalho complementa a taxonomia dos palinomorfos do perfil sedimentar publicada por Leonhardt \& Lorscheitter $(2007,2008)$. Nesta terceira parte são apresentados os grãos de pólen de Magnoliopsida (Asteridae) e Liliopsida (Alismatidae, Commelinidae e Liliidae), e de algumas angiospermas indeterminadas.

\section{Material e métodos}

A turfeira localiza-se no município de São Francisco de Paula (figura 1), em Alpes de São Francisco (29²9’ S$50^{\circ} 37^{\prime} \mathrm{W}$, altitude de $911 \mathrm{~m}$ ). A coleta do perfil sedimentar de $286 \mathrm{~cm}$, com o Amostrador de Hiller (Faegri \& Iversen 1989), foi realizada na porção central mais espessa da turfeira. Foram retiradas 22 amostras a intervalos regulares ao longo do perfil, cada uma contendo $8 \mathrm{~cm}^{3}$ de sedimento fresco. Uma datação por ${ }^{14} \mathrm{C}$ foi realizada em sedimentos do nível próximo à base do perfil $(273 \mathrm{~cm})$ pelo laboratório Beta Analytic Inc., Miami, Flórida, para determinar o intervalo de tempo geológico. Para a palinologia as amostras foram tratadas com $\mathrm{HCl}, \mathrm{HF}, \mathrm{KOH}$ e acetólise (Faegri \& Iversen 1989), com filtragem em malha de $250 \mu \mathrm{m}$ e montagem das lâminas em gelatina-glicerinada. A análise foi realizada ao fotomicroscópio óptico Diaplan Leitz, com objetivas planapocromáticas, em aumentos de 400 e $1000 \times$. As fotomicrografias foram feitas em aumento de $1000 \times$ (filme T-MAX 100).

Para a identificação botânica do conteúdo polínico foram utilizadas a palinoteca de plantas atuais do Laboratório de
Palinologia do Departamento de Botânica da Universidade Federal do Rio Grande do Sul e bibliografia especializada. As principais obras consultadas foram: Heusser 1971, Markgraf \& D'Antoni 1978, Ybert 1979, Wingenroth \& Heusser 1983, Lorscheitter 1988, Roubik \& Moreno 1991, Neves \& Lorscheitter 1995, Colinvaux et al. 1999. Na determinação, procurou-se sempre alcançar a categoria taxonômica mais inferior possível. Números foram acrescentados após os nomes dos grãos de pólen morfologicamente distintos, atribuídos a uma mesma categoria taxonômica. A palavra "Tipo" foi empregada antes dos nomes que não tiveram sua determinação garantida em função de semelhanças dentro de um mesmo grupo taxonômico, conforme o usual em palinologia de sedimentos (Berglund 1986). Quando a identificação não foi possível, o material recebeu uma denominação apenas morfológica.

As descrições dos grãos de pólen foram feitas visando uma caracterização sucinta. $\mathrm{O}$ eixo polar e o eixo equatorial foram medidos em ocular de fio móvel e as medidas convertidas para micrômetros. Grãos de pólen encontrados apenas na vista polar não puderam ser classificados quanto à forma. Dados ecológicos do esporófito de origem foram incluídos para subsidiar reconstituições paleoambientais. Os grãos de pólen são apresentados em ordenação sistemática, conforme Cronquist (1981).

\section{Resultados e discussão}

A datação radiométrica da base da seqüência revelou $24.930 \pm 180$ anos AP (Beta 194784), permitindo

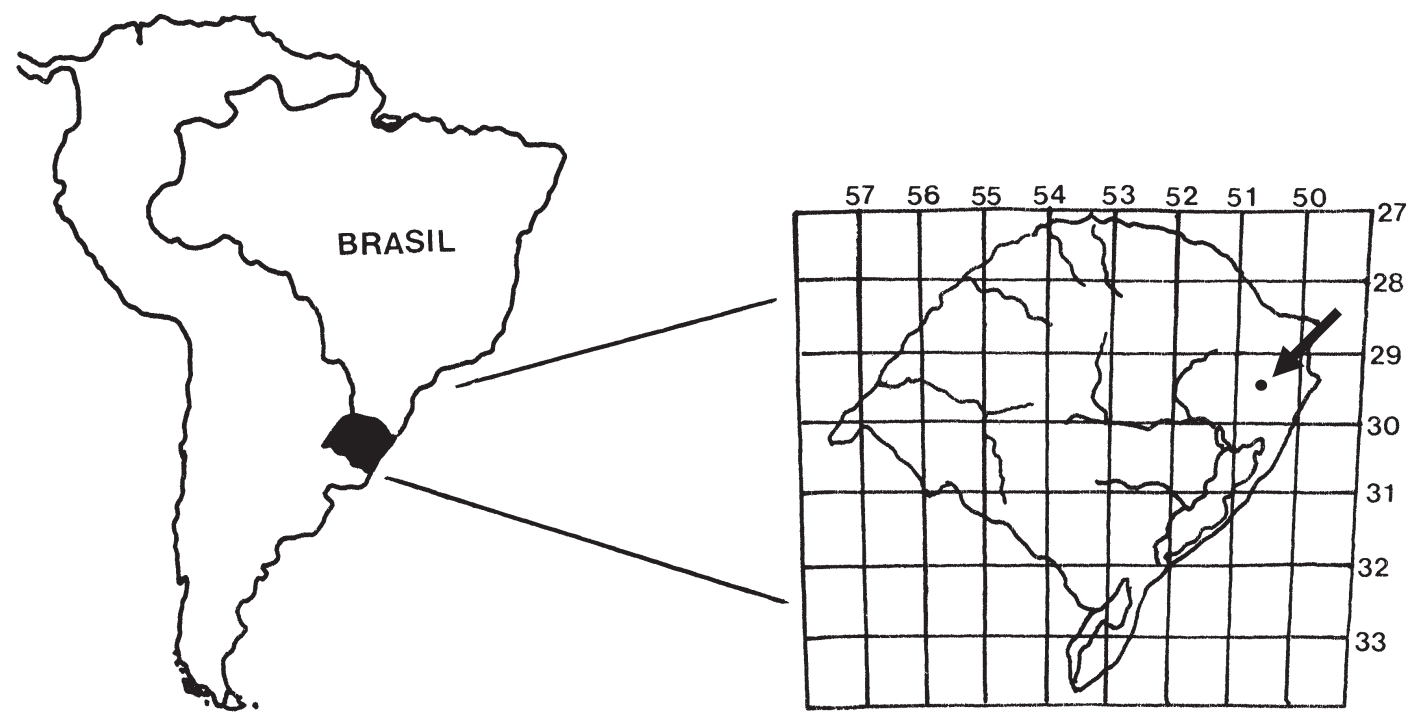

Figura 1. Estado do Rio Grande do Sul e a localização da turfeira estudada (•) a 29²9’ S e $50^{\circ} 37^{\prime}$ W, Município de São Francisco de Paula, Planalto Leste.

Figure 1. Rio Grande do Sul State and the studied bog (•) at $29^{\circ} 29^{\prime}$ S and $50^{\circ} 37^{\prime}$ W, São Francisco de Paula Municipality, eastern Plateau. 
determinar um intervalo de tempo que abrange o Pleistoceno Tardio e todo o Holoceno.

\section{Divisão Magnoliophyta \\ Classe Magnoliopsida \\ Subclasse Asteridae \\ Ordem Solanales \\ Família Convolvulaceae}

\section{Tipo Evolvulus L.}

Figuras 2-4.

Esférico, radiossimétrico, apolar. Pantocolpado, microequinado. Sincolpado, formando placas características. Columelas evidentes. Diâmetro do grão: cerca de $31 \mu \mathrm{m}$. Diâmetro das placas: cerca de $15 \mu \mathrm{m}$. Dados ecológicos: muitas espécies do gênero são comuns como lianas de bordas de florestas (Souza \& Lorenzi 2005).

Ordem Lamiales

Família Lamiaceae

\section{Lamiaceae}

Figuras 5-8.

Subprolato a perprolato, radiossimétrico, isopolar. Circular em vista polar, elíptico em vista equatorial. Estefanocolpado, per-reticulado, em geral com seis colpos. Colpos finos e longos. Retículo fino. Columelas evidentes, sustentando o muro. Eixo polar: $34-40 \mu \mathrm{m}$. Eixo equatorial: 19-26 $\mu \mathrm{m}$. Dados ecológicos: ervas anuais ou perenes, subarbustos ou arbustos, de ambientes variados. Distribuem-se pela América tropical (Barroso et al. 1991, Schultz 1984, Harley 1985).

\section{Tipo Lamiaceae}

Figuras 9-11.

Oblato-esferoidal, radiossimétrico, isopolar. Circular em vista equatorial. Estefanocolpado per-reticulado, com seis colpos. Colpos finos e longos. Retículo grosseiro. Columelas evidentes, sustentando o muro. Eixo polar: cerca de $31 \mu \mathrm{m}$. Eixo equatorial: cerca de $33 \mu \mathrm{m}$. Obs: diferencia-se de Lamiaceae pela forma do grão e pelo tamanho mais avantajado da malha do retículo. Dados ecológicos: como em Lamiaceae.

Família Verbenaceae

\section{Verbena L.}

Figuras 12-14.

Esférico a oblato, radiossimétrico, isopolar. Subtriangular em vista polar, com zonas interangulares um pouco convexas. Circular ou levemente elíptico em vista equatorial. Tricolporado, psilado, colporos com nítida margem espessada. Columelas evidentes. Eixo polar: cerca de $26 \mu \mathrm{m}$. Eixo equatorial: $25-35 \mu \mathrm{m}$. Obs: foram encontrados grãos provavelmente de duas espécies. Dependendo do grão as columelas podem ser observadas por transparência, como finas pontuações. Dados ecológicos: ervas campestres, comuns no Rio Grande do Sul (Schultz 1984).

Ordem Plantaginales

Família Plantaginaceae

\section{Plantago L.}

Figuras 15-17.

Esférico, radiossimétrico, apolar. Pantoporado, verrucado. Poros tênues e distanciados. Verrugas delicadas. Columelas evidentes. Diâmetro: 20-29 $\mu \mathrm{m}$. Dados ecológicos: cosmopolita, composto por plantas herbáceas campestres, preferentemente em zonas temperadas e frias (Schultz 1984). Com representantes no Planalto do Rio Grande do Sul (Rambo 1956).

\section{Ordem Scrophulariales \\ Família Bignoniaceae}

\section{Bignoniaceae}

Figuras 18-22.

Prolato, radiossimétrico, isopolar. Circular ou subtriangular com zonas interangulares levemente convexas em vista polar. Elíptico em vista equatorial. Tricolpado, per-reticulado. Colpos longos e largos. Retículo de tamanho variável. Columelas evidentes, sustentando o muro. Eixo polar: cerca de $54 \mu \mathrm{m}$. Eixo equatorial: 33-53 $\mu \mathrm{m}$. Dados ecológicos: plantas lenhosas, arbustivas ou arbóreas e também trepadeiras. Família essencialmente tropical, poucas espécies na zona temperada (Joly 2002, Barroso et al. 1991, Schultz 1984, Souza \& Lorenzi 2005). Com várias espécies no Planalto do Rio Grande do Sul (Rambo 1956).

Família Lentibulariaceae

\section{Utricularia L.}

Figuras 23-25.

Suboblato a oblato-esferoidal, radiossimétrico, isopolar. Aproximadamente circular em vista polar. Estefanocolporado, psilado. Colporos longos. Endoaberturas unidas, formando uma faixa contínua na região equatorial saliente. Estratificação obscura. Eixo polar: 20-22 $\mu \mathrm{m}$. Eixo equatorial: 23-24 $\mu \mathrm{m}$. Dados 

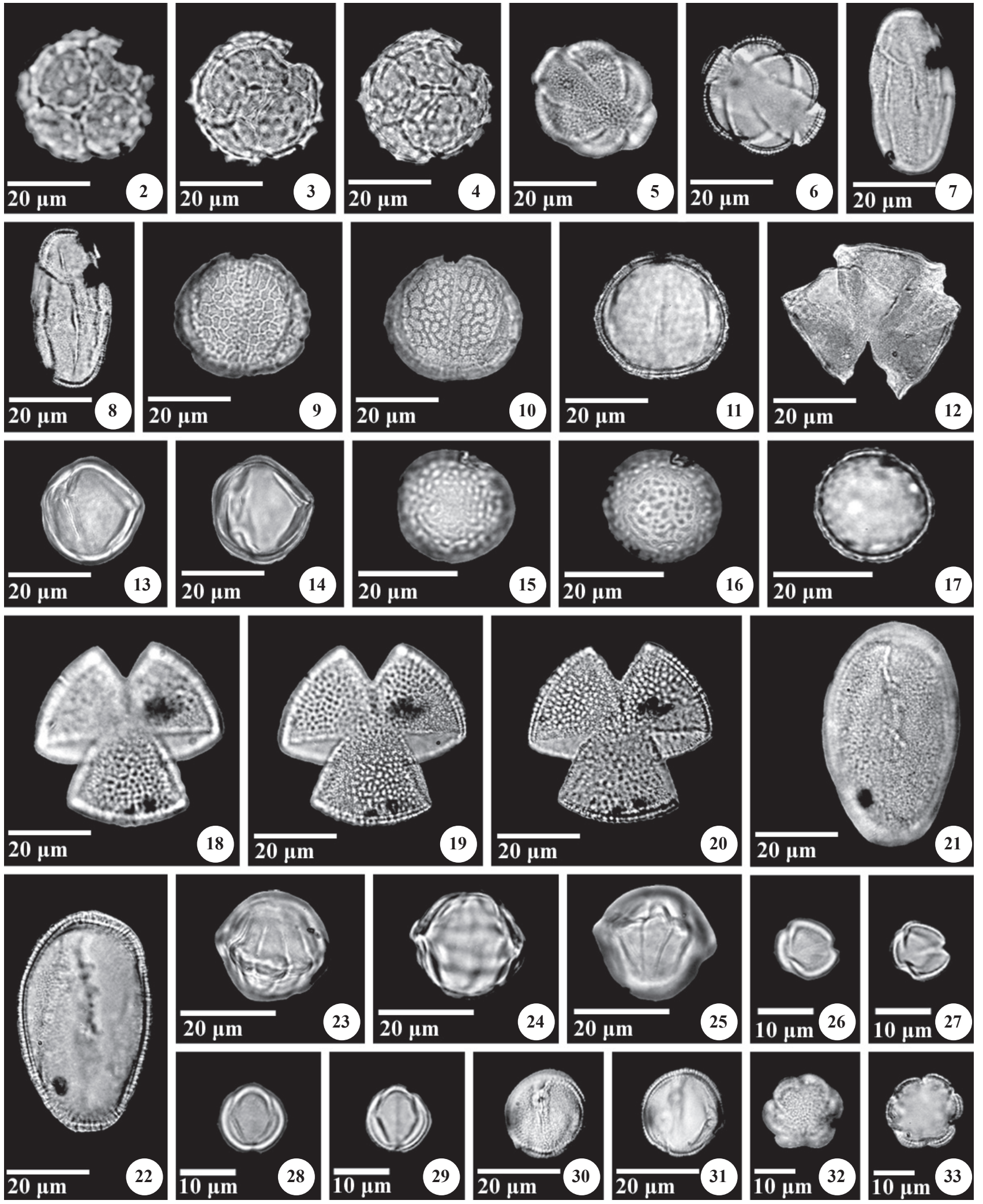

Figuras 2-33. Magnoliopsida. 2-4. Tipo Evolvulus L.: 1ํ-3opl. 5-8. Lamiaceae. 5-6 (P, oblíquo): 1o-2o pl., 7-8 (EQ): 1-2o pl. 9-11.

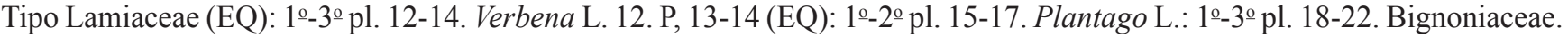
18-20 (P): 1--3ำ pl., 21-22 (EQ): 1--2o pl. 23-25. Utricularia L. (EQ, oblíquo): 1--3o pl. 26-29. Tipo Scrophulariaceae. 26-27

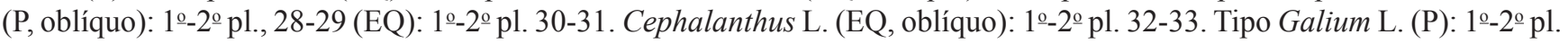
$(\mathrm{pl}=$ planos; $\mathrm{P}=$ vista polar; $\mathrm{EQ}=$ vista equatorial $)$. 
ecológicos: pequenas ervas heliófilas e higrófilas seletivas, ocorrendo preferencialmente sobre solos rochosos úmidos, banhados rasos e turfeiras (Taylor 1980).

Família Scrophulariaceae

\section{Tipo Scrophulariaceae}

Figuras 26-29.

Subprolato a prolato, muito pequeno. Radiossimétrico, isopolar. Subtriangular em vista polar, levemente elíptico em vista equatorial. Tricolporado, psilado. Estratificação obscura. Eixo polar: 10$18 \mu \mathrm{m}$. Eixo equatorial: 8-11 $\mu \mathrm{m}$. Dados ecológicos: a família compõe-se de plantas em geral herbáceas ou subarbustivas, mais raramente árvores. Cosmopolita, preferindo zonas temperadas. Na flora brasileira, várias espécies são campestres ou ruderais (Joly 2002, Barroso et al. 1991, Schultz 1984, Ichaso \& Barroso 1970).

Ordem Rubiales

Família Rubiaceae

\section{Cephalanthus L.}

Figuras 30-31.

Prolato-esferoidal, radiossimétrico, isopolar. Aproximadamente circular em vista equatorial. Tricolporado, per-reticulado. Endoaberturas com espessamento anelar marginal. Columelas evidentes, sustentando o muro. Eixo polar: cerca de $22 \mu \mathrm{m}$. Eixo equatorial: cerca de $20 \mu \mathrm{m}$. Dados ecológicos: plantas arbustivas, nativas de regiões tropicais e temperadas da América, África e Ásia (Delprete et al. 2004).

\section{Tipo Galium L.}

Figuras 32-33.

Radiossimétrico, isopolar, pequeno. Circular em vista polar. Estefanocolpado, psilado. Columelas evidentes. Eixo equatorial: 19-27 $\mu \mathrm{m}$. Obs: nome atual do gênero Relbunium (Endl.) Hook. f. Dados ecológicos: gênero de ervas anuais ou perenes, eretas, ou trepadeiras, muito raramente subarbustos, eretos ou escandentes. No Rio Grande do Sul ocorrem espécies de habitat variado, sobre campos úmidos ou não, borda de capões, margens de rios ou locais brejosos (Delprete et al. 2004).

\section{Rubiaceae 1}

Figuras 34-37.

Subprolato a suboblato, radiossimétrico, isopolar. Circular em vista polar, elíptico em vista equatorial. Estefanocolporado, psilado. Colporos longos. Endoaberturas grandes, com espessamento marginal da exina. Columelas evidentes. Eixo polar: $36-40 \mu \mathrm{m}$. Eixo equatorial: $31-47 \mu \mathrm{m}$. Dados ecológicos: árvores, arbustos, ervas eretas ou rastejantes, trepadeiras volúveis herbáceas ou lenhosas, ou lianas, terrícolas ou raramente epifíticas. Ocorrem especialmente em zonas tropicais e subtropicais, com poucas espécies em zonas temperadas e frias (Delprete et al. 2004).

\section{Rubiaceae 2}

Figuras 38-40.

Radiossimétrico, isopolar. Aproximadamente circular em vista polar. Estefanocolpado, psilado. Colpos curtos. Columelas evidentes. Eixo equatorial: cerca de $30 \mu \mathrm{m}$. Obs: columelas podem ser observadas por transparência, como finas pontuações. Dados ecológicos: Como em Rubiaceae 1.

\section{Rubiaceae 3}

Figuras 41-42.

Oblato-esferoidal, radiossimétrico, isopolar. Levemente elíptico em vista equatorial. Estefanocolporado, microequinado. Colporos curtos. Endoaberturas grandes, com espessamento marginal da exina. Columelas evidentes. Eixo polar: cerca de $42 \mu \mathrm{m}$. Eixo equatorial: cerca de $45 \mu \mathrm{m}$. Dados ecológicos: Como em Rubiaceae 1.

\section{Ordem Dipsacales \\ Família Valerianaceae}

\section{Valeriana L.}

Figuras 43-48.

Prolato-esferoidal, radiossimétrico, isopolar. Circular em vista polar, circular, elíptico em vista equatorial. Tricolpado, microequinado. Espinhos finos, distanciados, regularmente distribuídos. Columelas evidentes. Eixo polar: cerca de $32 \mu \mathrm{m}$. Eixo equatorial: 28-34 $\mu \mathrm{m}$. Dados

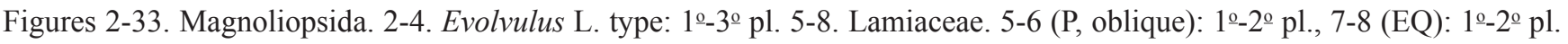
9-11. Lamiaceae type (EQ): 1ํ-3ำ pl. 12-14. Verbena L. 12 (P), 13-14 (EQ): 1-2º pl. 15-17. Plantago L.: 1-3 pl. 18-22.

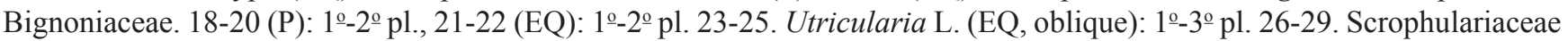

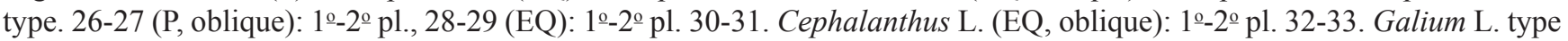
$(\mathrm{P}): 1^{\circ}-2^{\circ} \mathrm{pl} .(\mathrm{pl}=$ planes; $\mathrm{P}=$ polar view; $\mathrm{EQ}=$ equatorial view $)$. 

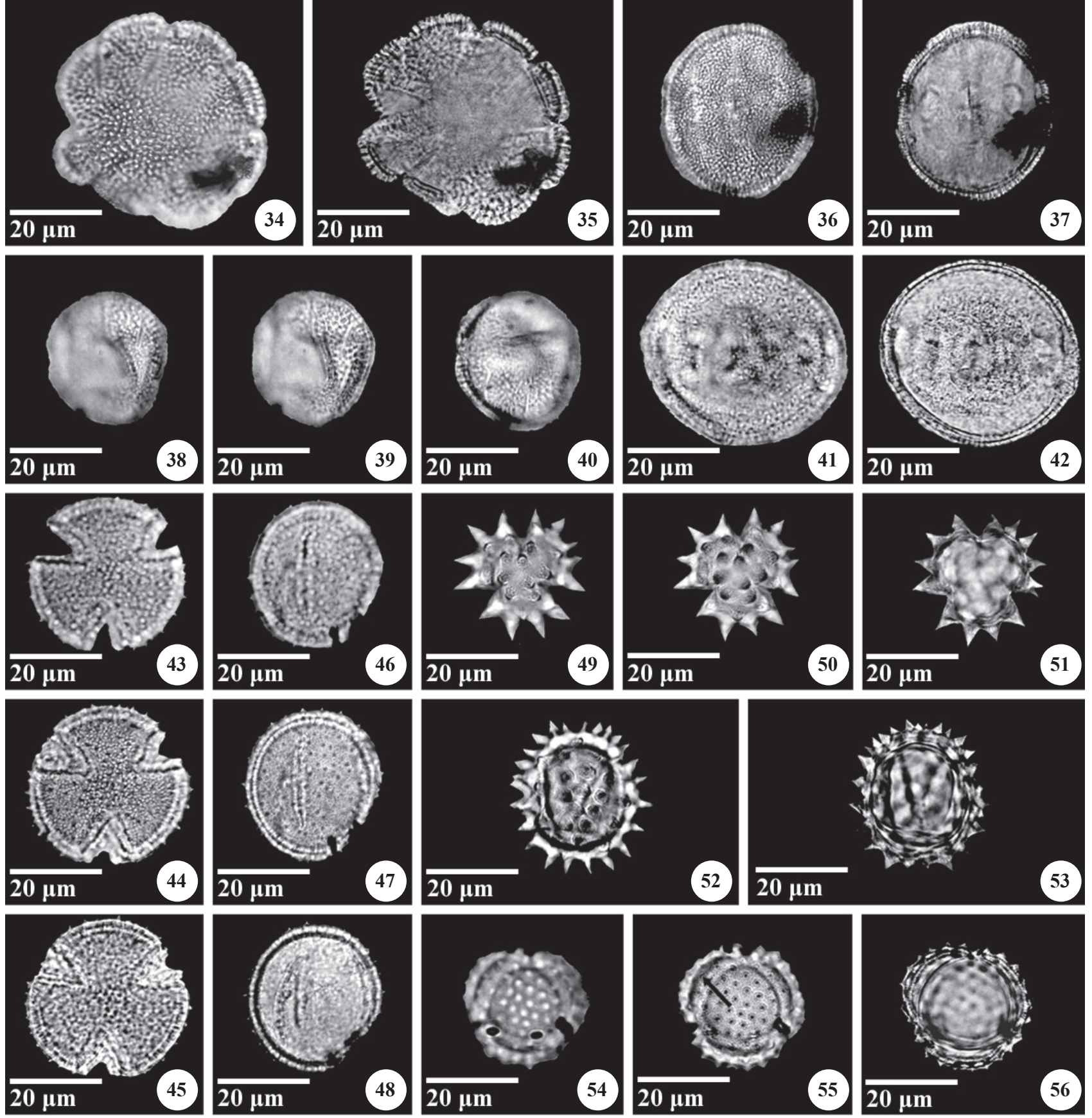

(41)
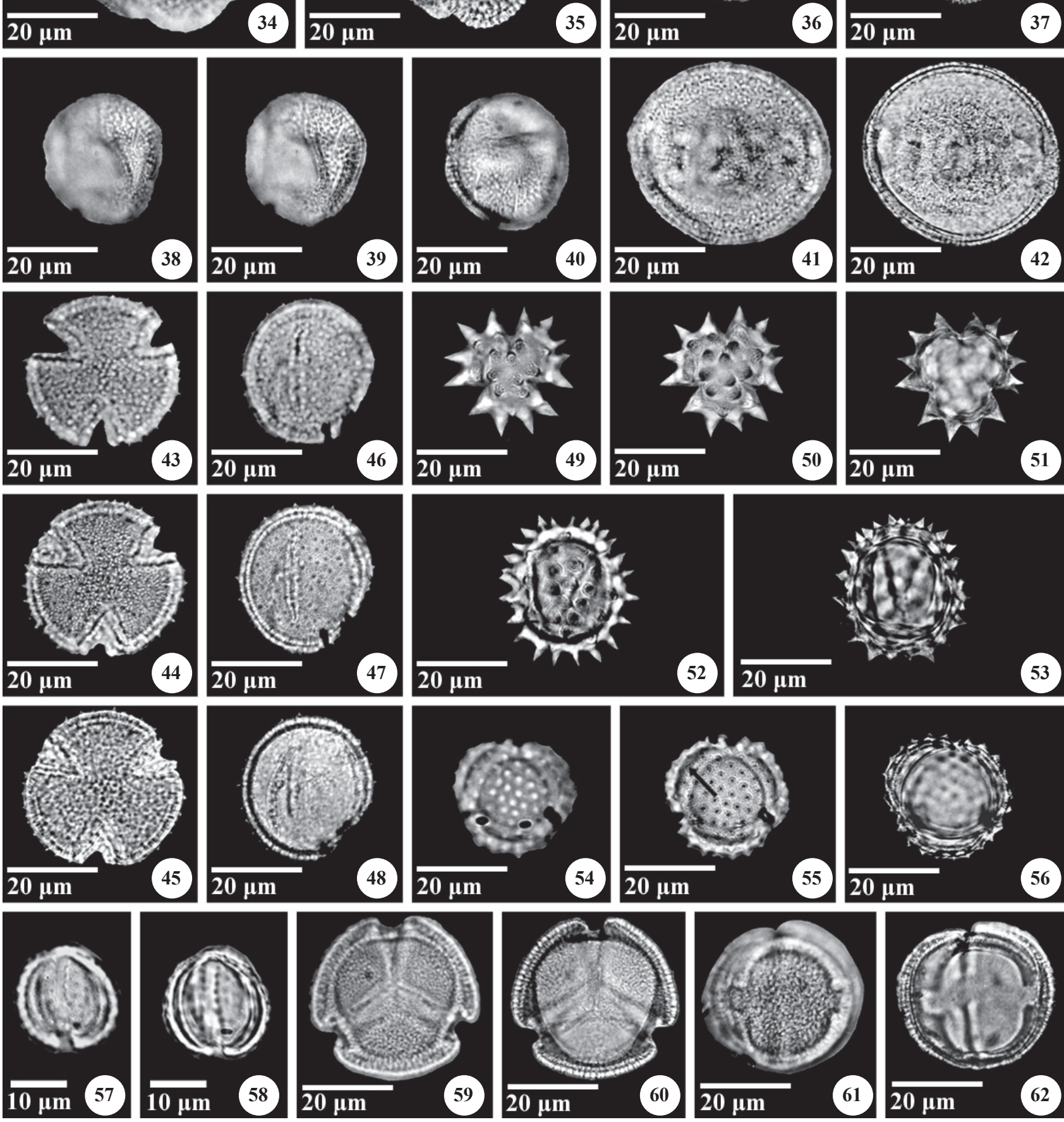

53
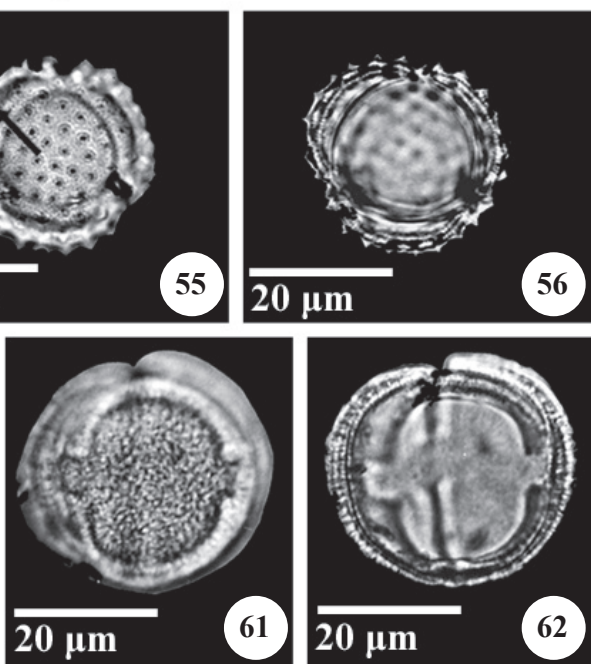

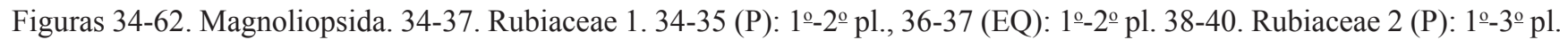

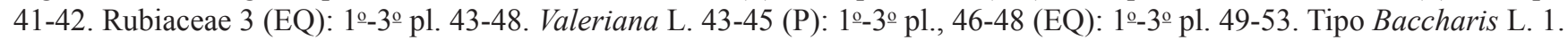
49-51 (P): 1--3ำ pl., 52-53 (EQ): 1-2o pl. 54-56. Tipo Baccharis L. 2 (P): 1--3ำ pl., 55. Cava evidente (seta), 57-58. Tipo

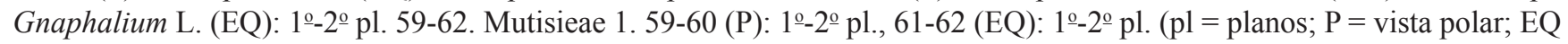
$=$ vista equatorial). 
ecológicos: ervas ou subarbustos, raramente trepadeiras. Ocorrem em campos rupestres, interior e margem de florestas, margem de matas de galeria e banhados. Ampla dispersão no Rio Grande do Sul (Sobral 1999).

Ordem Asterales

Família Asteraceae

15. Tipo Baccharis L. 1

Figuras 49-53.

Prolato-esferoidal, radiossimétrico, isopolar. Circular em vista polar, circular a elíptico em vista equatorial. Tricolporado, equinado. Espinhos longos, regularmente distribuídos. Columelas evidentes. Eixo polar: 26-32 $\mu \mathrm{m}$. Eixo equatorial: 24-29 $\mu \mathrm{m}$. Dados ecológicos: o gênero está composto por ervas e arbustos especialmente campestres (Joly 2002). Várias espécies destacam-se nos campos do Planalto do Rio Grande do Sul (Rambo 1956).

\section{Tipo Baccharis L. 2}

Figuras 54-56.

Radiossimétrico, isopolar. Circular em vista polar. Tricolporado, equinado. Espinhos curtos, regularmente distribuídos. Columelas evidentes. Descolamento da nexina evidente, formando uma cava entre a nexina 1 e a nexina 2. Eixo equatorial: cerca de $27 \mu \mathrm{m}$. Dados ecológicos: como em Tipo Baccharis 1.

\section{Tipo Gnaphalium L.}

Figuras 57-58.

Prolato-esferoidal, radiossimétrico, isopolar. Circular em vista polar e equatorial. Tricolporado, microequinado. Espinhos regularmente dispostos. Columelas evidentes. Eixo polar: cerca de $19 \mu \mathrm{m}$. Eixo equatorial: cerca de $18 \mu \mathrm{m}$. Dados ecológicos: o gênero é composto por ervas comuns na região litorânea e em terrenos alterados (Joly 2002). Gênero presente nos campos do Planalto do Rio Grande do Sul (Rambo 1956).

\section{Mutisieae 1}

Figuras 59-62.

Oblato-esferoidal a prolato-esferoidal, radiossimétrico, isopolar. Circular em vista polar, levemente elíptico em vista equatorial. Tricolporado, rugulado. Colporos longos, com endoaberturas grandes. Exina biestratificada. camada externa um pouco mais espessa do que a interna. Columelas evidentes. Eixo polar: 31-33 $\mu \mathrm{m}$. Eixo equatorial: 30-37 $\mu \mathrm{m}$. Dados ecológicos: tribo constituída predominantemente por er vas e arbustos, mas também lianas e árvores. $\mathrm{O}$ maior número de espécies da tribo tem formas de vida relacionadas predominantemente a ambientes campestres (Mondim 1996).

\section{Mutisieae 2}

Figuras 63-64.

Subprolato a prolato, radiossimétrico, isopolar. Elíptico-fusiforme em vista equatorial, com pólos levemente projetados. Tricolporado, psilado. Colporos longos. Exina biestratificada, camada interna mais espessa na zona equatorial, tornando-se mais fina que a camada externa nos pólos. Columelas evidentes. Eixo polar: 46-52 $\mu \mathrm{m}$. Eixo equatorial: 33-36 $\mu \mathrm{m}$. Obs: columelas podem ser observadas por transparência, como finas pontuações. Dados ecológicos: como em Mutisieae 1.

\section{Mutisieae 3}

Figuras 65-66.

Subprolato, radiossimétrico, isopolar. Elípticofusiforme em vista equatorial. Tricolporado, psilado. Colporos longos. Columelas evidentes em sexina biestratificada, camada interna mais espessa do que a externa. Eixo polar: 37-47 $\mu \mathrm{m}$. Eixo equatorial: $30-$ $36 \mu \mathrm{m}$. Obs: columelas podem ser observadas por transparência, como finas pontuações. Dados ecológicos: como em Mutisieae 1.

\section{Mutisieae 4}

Figuras 67-69.

Prolato, radiossimétrico, isopolar. Elíptico em vista equatorial. Tricolporado, equinado. Colporos longos. Espinhos pequenos, regularmente distribuídos. Columelas evidentes. Eixo polar: 36-44 $\mu \mathrm{m}$. Eixo equatorial: $24-$ $29 \mu \mathrm{m}$. Dados ecológicos: Como em Mutisieae 1. Obs: as diferenças entre Mutisieae 1, Mutisieae 2, Mutisieae 3 e Mutisieae 4 são a forma, o tipo de ornamentação e a distribuição das columelas.

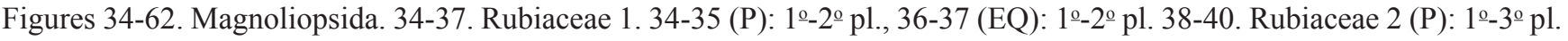
41-42. Rubiaceae 3 (EQ): 1-3o pl. 43-48. Valeriana L. 43-45 (P): 1-3o pl., 46-48 (EQ): 1-3 pl. 49-53. Baccharis L. 1 type. 49-51 (P): 1-3o pl., 52-53. (EQ): 1-2o pl. 54-56. Baccharis L. 2 type. (P): 1-3o pl., 55. Evident cavea (arrow), 57-58.

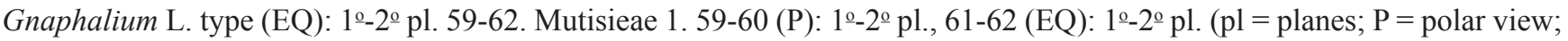
$\mathrm{EQ}=$ equatorial view). 

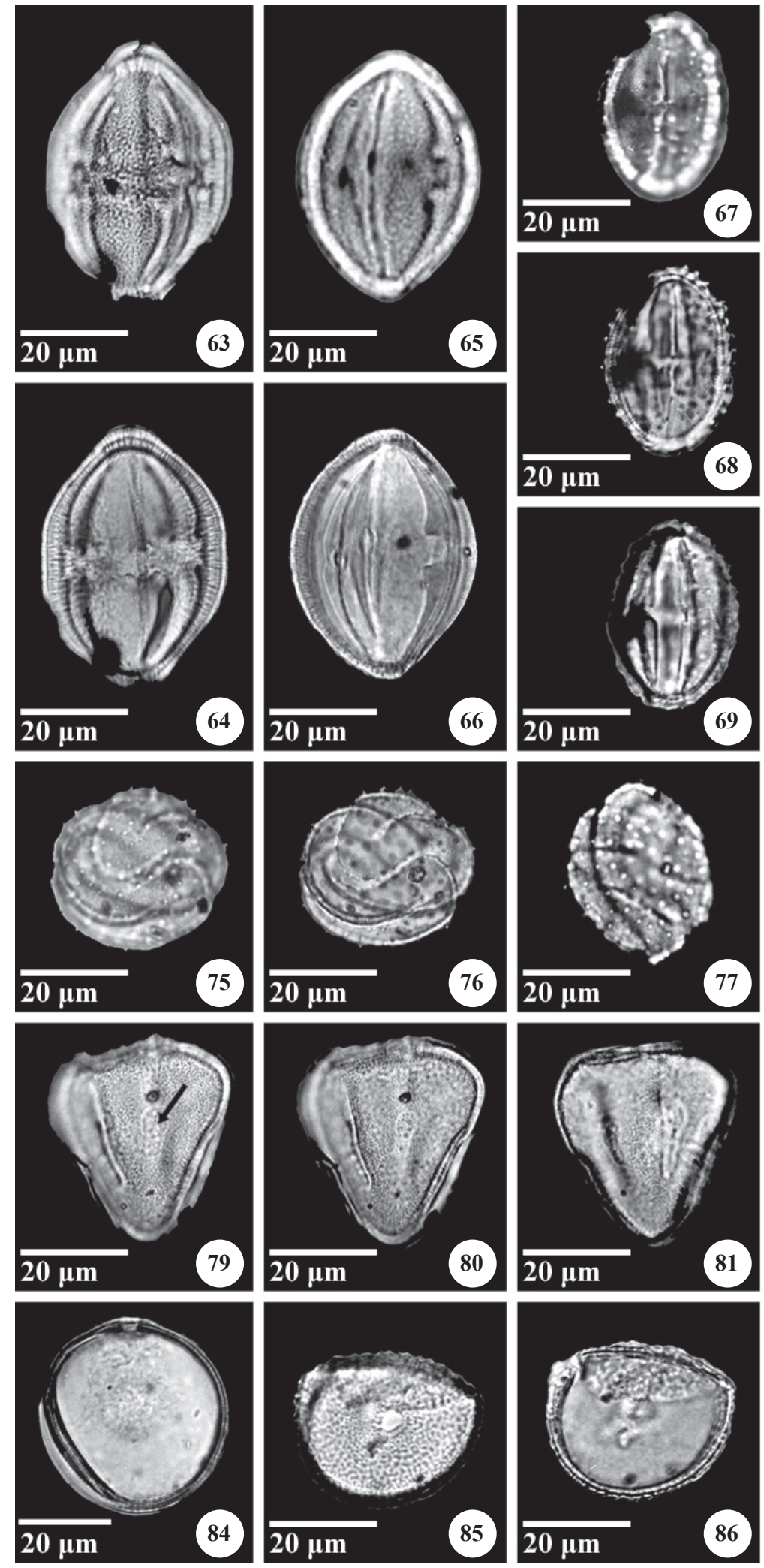
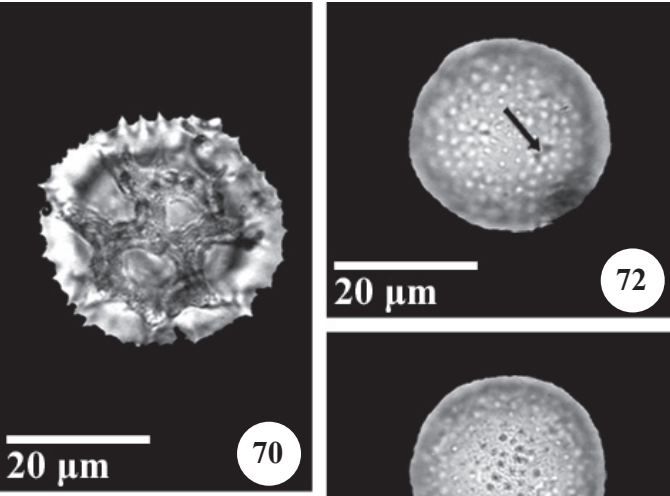

$20 \mu \mathrm{m}$

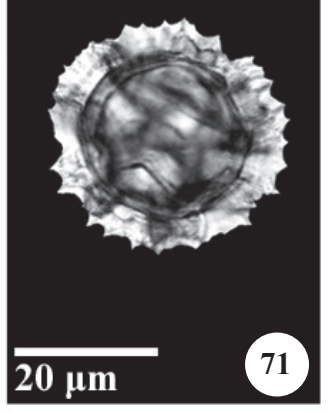

\section{$20 \mu \mathrm{m}$}
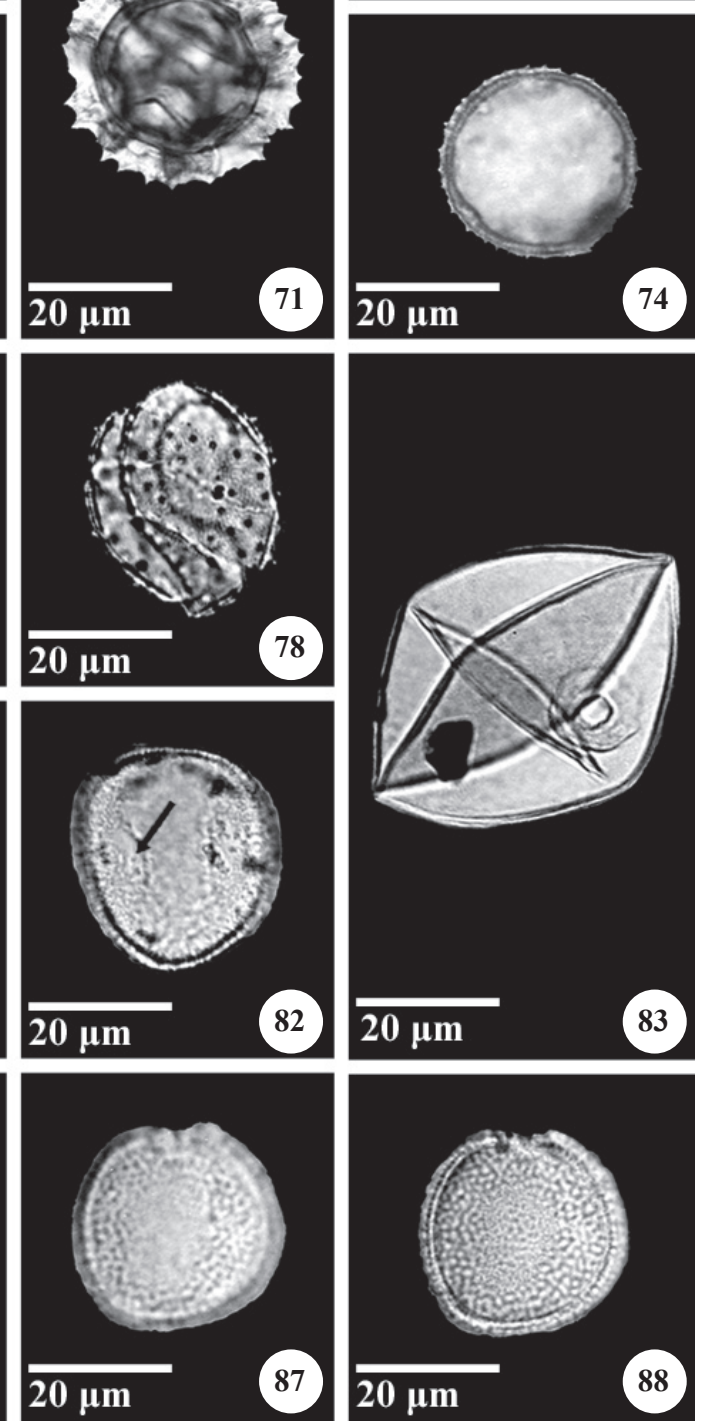

Figuras 63-71. Magnoliopsida. 63-64. Mutisieae 2 (EQ): 1-2o pl. 65-66. Mutisieae 3 (EQ): 1으으 pl. 67-69. Mutisieae 4 (EQ):

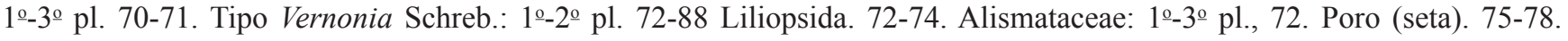

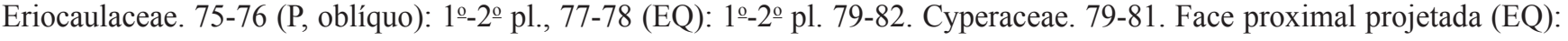
1-3o pl., 79. Sulco (seta), 82. Forma elíptica (EQ), Sulco (seta). 83-84. Poaceae. 83 (PD), 84 (EQ). 85-88. Typha L. 85-86 (PD,

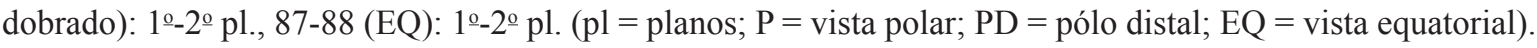




\section{Tipo Vernonia Schreb.}

Figuras 70-71.

Esférico, radiossimétrico, isopolar. Tricolporado, per-reticulado, equinado. Lofado, muro muito alto portando espinhos grandes, regularmente distribuídos, mascarando as aberturas. Columelas evidentes, sustentando o muro. Diâmetro: cerca de $28 \mu \mathrm{m}$. Dados ecológicos: o gênero está composto por ervas, subarbustos, arbustos e árvores que vivem em distintos ambientes como campos, banhados, capoeiras, borda e interior de matas e margem de rios, ocorrendo também como ruderais (Matzenbacher \& Mafioleti 1994).

\section{Classe Liliopsida \\ Subclasse Alismatidae \\ Ordem Alismatales \\ Família Alismataceae}

\section{Alismataceae}

Figuras 72-74.

Esférico, radiossimétrico, apolar. Pantoporado, microequinado. Poros tênues e distanciados. Espinhos muito finos, regularmente distribuídos. Columelas evidentes. Diâmetro: 26-30 $\mu \mathrm{m}$. Dados ecológicos: plantas herbáceas, aquáticas ou palustres, habitando zonas quentes e temperadas (Schultz 1984).

\section{Subclasse Commelinidae \\ Ordem Eriocaulales \\ Família Eriocaulaceae}

\section{Eriocaulaceae}

Figuras 75-78.

Oblato-esferoidal, radiossimétrico, isopolar. Espiraperturado, microequinado. Espinhos muito finos, distanciados, regularmente distribuídos. Columelas evidentes. Eixo polar: cerca de $28 \mu \mathrm{m}$. Eixo equatorial: cerca de $30 \mu \mathrm{m}$. Dados ecológicos: plantas herbáceas, heliófilas e higrófilas seletivas, em banhados ou campos úmidos (Moldenke \& Smith 1976). Freqüentes em turfeiras do Planalto
Leste, são também citadas por Rambo (1956) para esta região.

\section{Ordem Cyperales \\ Família Cyperaceae}

\section{Cyperaceae}

Figuras 79-82.

Prolato-esferoidal a subprolato, radiossimétrico, heteropolar. Pólo distal em geral alargado, afilando em direção ao pólo proximal. Inaperturado, monoulcerado com o ulcus no pólo distal, e/ou sulcado com um número variável de sulcos irregulares na região equatorial. Escabrado, ornamentação mais grosseira nas aberturas. Columelas nem sempre evidentes. Eixo polar: 38-48 $\mu \mathrm{m}$. Eixo equatorial: 35-39 $\mu \mathrm{m}$. Obs: grãos de pólen freqüentemente com dobras irregulares devido à fina espessura da exina. Dados ecológicos: plantas herbáceas, cosmopolitas, preferindo terrenos brejosos ou alagadiços (Joly 2002, Schultz 1984, Souza \& Lorenzi 2005). Muitas espécies no Planalto do Rio Grande do Sul (Rambo 1956).

\section{Família Poaceae}

\section{Poaceae}

Figuras 83-84.

Aproximadamente esférico, radiossimétrico, heteropolar. Circular em vista polar e equatorial. Monoporado, psilado a escabrado. Poro no pólo distal, com nítido espessamento anelar. Columelas evidentes. Eixo polar: $23-43 \mu \mathrm{m}$. Eixo equatorial: $24-41 \mu \mathrm{m}$. Obs: grãos freqüentemente apresentando dobras irregulares devido à fina espessura da exina. Dados ecológicos: plantas na grande maioria herbáceas, anuais ou perenes, predominando em ambientes abertos. Formadoras de campos, principalmente anemófilas. Apenas as espécies da subfamília Bambusoideae e afins habitam florestas, algumas delas apresentando adaptações para a entomofilia. Com aproximadamente 110 gêneros e 450 espécies no Rio Grande do Sul (Boldrini et al. 2005).

Figures 63-71. Magnoliopsida. 63-64. Mutisieae 2 (EQ): 1-2o pl. 65-66. Mutisieae 3 (EQ): 1ำ2o pl. 67-69. Mutisieae 4

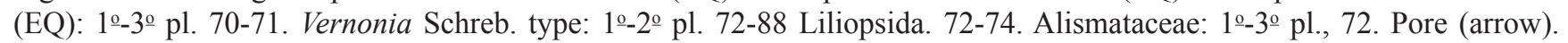
75-78. Eriocaulaceae. 75-76 (P, oblique): 1은 pl., 77-78 (EQ): 1은 pl. 79-82. Cyperaceae. 79-81. Protruding proximal face (EQ): 1-3ำ pl., 79. Sulcus (arrow), 82. Elliptical grain (EQ), showing a sulcus (arrow). 83-84. Poaceae. 83 (PD), 84 (EQ).

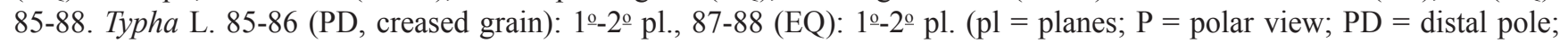
$\mathrm{EQ}=$ equatorial view). 

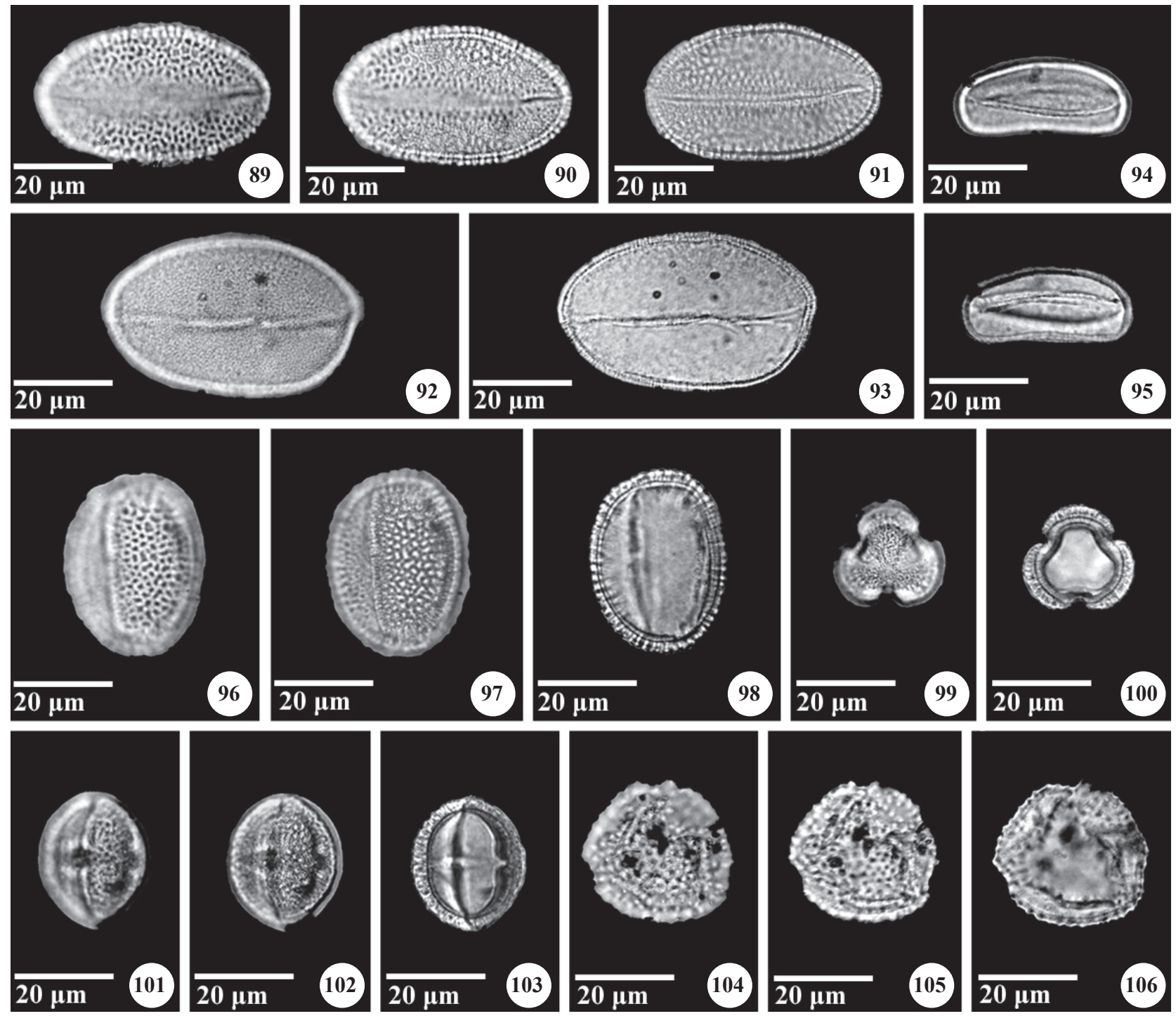

Figuras 89-95. Liliopsida. 89-91. Tipo Liliaceae 1 (PP): 1ํ-3ํㅡ. pl. 92-93. Tipo Liliaceae 2 (PP): 1ํ-2oㅡ. 94-95. Eichhornia (EQ):

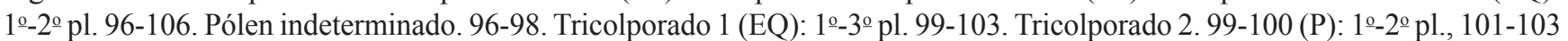
(EQ): $1^{\circ}-3^{\circ} \mathrm{pl}$. 104-106. Triporado $1(\mathrm{P})$ : $1^{\circ}-3^{\circ} \mathrm{pl}$. $(\mathrm{pl}=$ planos; $\mathrm{P}=$ vista polar; $\mathrm{PP}=$ pólo proximal; $\mathrm{EQ}=$ vista equatorial).

Figures 89-95. Liliopsida. 89-91. Liliaceae 1 type (PP): $1^{\circ}-3 \circ-\mathrm{pl}$. 92-93. Liliaceae 2 type (PP): $1^{\circ}-2^{\circ} \mathrm{pl}$. 94-95. Eichhornia (EQ):

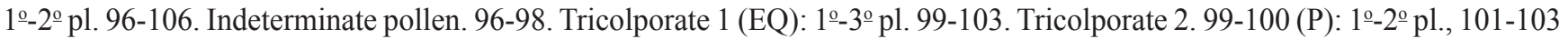

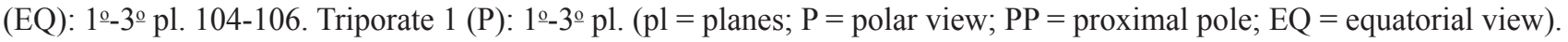

Ordem Typhales

Família Typhaceae

\section{Typha L.}

Figuras 85-88.

Suboblato a oblato-esferoidal, radiossimétrico, heteropolar. Aproximadamente circular em vista polar e equatorial. Monoulcerado, reticulado-rugulado, com ulcus no pólo distal. Columelas evidentes. Eixo polar: cerca de $28 \mu \mathrm{m}$. Eixo equatorial: 29-36 $\mu \mathrm{m}$. Dados ecológicos: ervas aquáticas, palustres ou lacustres (Reitz 1984).
Subclasse Liliidae

Ordem Liliales

Família Liliaceae

\section{Tipo Liliaceae 1}

Figuras 89-91.

Bilateral, heteropolar. Elíptico em vista polar. Monossulcado, per-reticulado. Sulco longo no pólo distal. Retículo desenvolvido. Columelas evidentes, sustentando o muro. Eixo equatorial maior: 35-52 $\mu \mathrm{m}$. Eixo equatorial menor: 28-38 $\mu \mathrm{m}$. Dados ecológicos: a 
família está composta por plantas geralmente herbáceas, perenes, cosmopolitas. Ocorre em diversos tipos de ambientes (Joly 2002, Schultz 1984).

29. Tipo Liliaceae 2

Figuras 92-93.

Bilateral, heteropolar, elíptico em vista polar. Monossulcado, per-reticulado. Sulco longo no pólo distal. Microrreticulado. Columelas evidentes sustentando o muro. Eixo equatorial maior: cerca de $52 \mu \mathrm{m}$. Eixo equatorial menor: 27-33 $\mu \mathrm{m}$. Obs: Tipo Liliaceae 2 difere do Tipo Liliaceae 1 pelo tamanho muito pequeno da malha do retículo. Dados ecológicos: como os do Tipo Liliaceae 1.

Família Pontederiaceae

\section{Eichhornia Kunth}

Figuras 94-95.

Peroblato, bilateral, heteropolar. Plano-convexo em vista equatorial. Bissulcado, psilado. Sulcos longos. Estratificação obscura. Eixo polar: cerca de $15 \mu \mathrm{m}$. Eixo equatorial: cerca de $35 \mu \mathrm{m}$. Dados ecológicos: plantas aquáticas, comuns em rios, riachos e lagos (Schultz 1984, Souza \& Lorenzi 2005).

Pólen indeterminado de Angiospermas

\section{Tricolporado 1}

Figuras 96-98.

Subprolato, radiossimétrico, isopolar. Elíptico em vista equatorial. Tricolporado per-reticulado. Colporos longos, retículo desenvolvido. Columelas evidentes, sustentando o muro. Eixo polar: cerca de $37 \mu \mathrm{m}$. Eixo equatorial: cerca de $28 \mu \mathrm{m}$.

\section{Tricolporado 2}

Figuras 99-103.

Prolato-esferoidal a subprolato, radiossimétrico, isopolar. Subtriangular em vista polar, elíptico em vista equatorial. Tricolporado per-reticulado. Colporos longos. Columelas evidentes. Sexina nitidamente mais espessa do que a nexina, afilando nos pólos. Eixo polar: 24-27 $\mu \mathrm{m}$. Eixo equatorial: $21-23 \mu \mathrm{m}$.

\section{Triporado 1}

Figuras 104-106.

Radiossimétrico, isopolar, aproximadamente subtriangular em vista polar. Triporado, microequinado. Poros tênues. Espinhos finos, densamente distribuídos. Columelas evidentes. Eixo equatorial: cerca de $27 \mu \mathrm{m}$.
Ao longo do perfil sedimentar foi encontrado material polínico de 22 Asteridae, uma Alismatidae, quatro Commelinidae, três Liliidae, além de pólen indeterminado de três angiospermas. O Tipo Evolvulus L. é citação nova para o Quaternário do Rio Grande do Sul.

Agradecimentos - as autoras agradecem ao CNPq e à Capes pelas bolsas e auxílios financeiros concedidos, que tornaram possível a realização deste trabalho.

\section{Referências bibliográficas}

BARROSO, G.M., GUIMARÃES, E.F., ICHASO, C.L.F., COSTA, C.G., PEIXOTO, A.L. \& LIMA, H.C. 1991. Sistemática de Angiospermas do Brasil. v.3. Imprensa Universitária, Universidade Federal de Viçosa, Viçosa.

BEHLING, H., BAUERMANN, S. \& NEVES, P.C.P. 2001. Holocene environmental changes in the São Francisco de Paula region, southern Brazil. Journal of South American Earth Sciences 14:631-639.

BEHLING, H., PILLAR, V.P., ORLÓCI,L. \& BAUERMANN, S.G. 2004. Late Quaternary Araucaria forest, grasslands (Campos), fire and climate dynamics, studied by highresolution pollen, charcoal and multivariate analysis of the Cambará do Sul core in southern Brazil. Palaeogeography, Palaeoclimatology, Palaeoecology 203:277-297.

BERGLUND, B.E. 1986. Handbook of Holocene palaeoecology and palaeohydrology. John Wiley \& Sons, New York.

BOLDRINI, I.I., LONGHI-WAGNER, H.M. \& BOECHAT, S.C. 2005. Morfologia e taxonomia de gramíneas sul-rio-grandenses. Editora da Universidade/UFRGS, Porto Alegre.

COLINVAUX, P., OLIVEIRA, P.E.D. \& PATIÑO, J.E.M. 1999. Amazon pollen manual and atlas. Harwood Academic Publishers, Amsterdam.

CRONQUIST, A. 1981. An integrated system of classification of flowering plants. Columbia University Press, New York.

DELPRETE, P.G., SMITH, L.B. \& KLEIN, R.M. 2004. Rubiáceas (gêneros de A-G). In Flora Ilustrada Catarinense (R. Reitz, ed.). Herbário Barbosa Rodrigues, Itajaí, p.1-345.

FAEGRI, K. \& IVERSEN, J. 1989. Textbook of pollen analysis. John Wiley \& Sons, New York.

HARLEY, R.M. 1985. Labiadas. In Flora Ilustrada Catarinense (R. Reitz, ed.). Herbário Barbosa Rodrigues, Itajaí, p.1-72.

HEUSSER, C.J. 1971. Pollen and spores of Chile. The University of Arizona Press, Tucson.

ICHASO, C.L.F. \& BARROSO, G.M. 1970. Escrofulariáceas. In Flora Ilustrada Catarinense (R. Reitz, ed.). Herbário Barbosa Rodrigues, Itajaí, p.1-114. 
JOLY, A.B. 2002. Botânica: introdução à taxonomia vegetal. 13a ed. Editora Nacional, São Paulo.

KLEIN, R.M. 1975. Southern Brazilian phytogeographic features and the probable influence of upper Quaternary climatic changes in the floristic distribution. Boletim Paranaense de Geociências 33:67-88.

LEONHARDT, A. \& LORSCHEITTER, M.L. 2007. Palinomorfos do perfil sedimentar de uma turfeira em São Francisco de Paula, Planalto Leste do Rio Grande do Sul, Sul do Brasil. Revista Brasileira de Botânica 30:47-59.

LEONHARDT, A. \& LORSCHEITTER, M.L. 2008. Pólen de gimnospermas e angiospermas do perfil sedimentar de uma turfeira em São Francisco de Paula, Planalto Leste do Rio Grande do Sul, Sul do Brasil. Revista Brasileira de Botânica 31:645-658.

LORSCHEITTER, M.L. 1988. Palinologia de sedimentos quaternários do testemunho T15, Cone de Rio Grande, Atlântico Sul, Brasil. Descrições Taxonômicas. Pesquisas 21:61-117.

MARKGRAF, V. \& D'ANTONI, H.L. 1978. Pollen flora of Argentina. The University of Arizona Press, Tucson.

MATZENBACHER, N.I. \& MAFIOLETI, S.I. 1994. Estudo taxonômico do gênero Vernonia Schreb. (Asteraceae) no Rio Grande do Sul, Brasil. Comunicações do Museu de Ciências e Tecnologia da PUCRS, série Botânica 1:1-133.

MOLDENKE, H.N. \& SMITH, L.B. 1976. Eriocauláceas. In Flora Ilustrada Catarinense (R. Reitz, ed.). Herbário Barbosa Rodrigues, Itajaí, p.1-103.

MONDIN, C.A. \& BAPTISTA, L.R.M. 1996. Relações biogeográficas da tribo Mutisieae Cass. (Asteraceae), sensu Cabrera, no Rio Grande do Sul. Comunicações do Museu Ciência Tecnologia da PUCRS, série Botânica 2:49-152.

NEVES, P.C.P. \& LORSCHEITTER, M.L. 1995. Palinologia de sedimentos de uma mata tropical paludosa (Terra de Areia, Planície Costeira Norte, Rio Grande do Sul, Brasil). Descrições Taxonômicas - Parte II: gimnospermas e angiospermas. Acta Geologica Leopoldensia 18:45-82.
NIMER, E. 1989. Climatologia do Brasil. 2a ed. Instituto Brasileiro de Geografia e Estatística, Rio de Janeiro.

RAMBO, B. 1953. História da flora do planalto rio-grandense. Sellowia 5:185-232.

RAMBO, B. 1956. A flora fanerogâmica dos Aparados riograndenses. Sellowia 7:235-298.

RAMBO, B. 2000. A fisionomia do Rio Grande do Sul ensaio de monografia natural. $3 \underline{a}$ ed. Ed. Universidade do Vale do Rio dos Sinos - Unisinos, São Leopoldo.

REITZ, R. 1984. Tifáceas. In Flora Ilustrada Catarinense (R. Reitz, ed.). Herbário Barbosa Rodrigues, Itajaí, p.1-16.

ROTH, L. \& LORSCHEITTER, M.L. 1993. Palynology of a bog in Parque Nacional de Aparados da Serra, East Plateau of Rio Grande Sul, Brazil. Quaternary of South America and Antarctic Peninsula 8:39-69.

ROUBIK, D.W. \& MORENO P.J.H. 1991. Pollen and spores of Barro Colorado Island. Monograph in Systematic Botany of the Missouri Botanical Garden 36:1-268.

SCHULTZ, A.R. 1984. Introdução ao estudo da botânica sistemática. Globo, Porto Alegre, v.2.

SOBRAL, M. 1999. Valerianaceae. Boletim do Instituto de Biociências - Flora Ilustrada do Rio Grande do Sul 58:1-61.

SOUZA, V.C. \& LORENZI, H. 2005. Botânica sistemática: guia ilustrado para identificação das famílias de Angiospermas da flora brasileira, baseado em APG II. Instituto Plantarum, Nova Odessa.

TAYLOR, P.G. 1980. Lentibulariáceas. In Flora Ilustrada Catarinense (R. Reitz, ed.). Herbário Barbosa Rodrigues, Itajaí, p.1-52.

VELOSO, H.P. 1962. Os grande clímaces do Brasil I Considerações sobre os tipos vegetativos da região sul. Memórias do Instituto Oswaldo Cruz 60:175-189.

WINGENROTH, M. \& HEUSSER, C.J. 1983. Pollen of the High Andean Flora. Ianigla, Mendoza.

YBERT, J.P. 1979. Atlas de pollens de Cote d'Ivoire. Office de La Recherche Scientifique et Technique Outre-mer, Paris. 